\title{
Arae SaCrae
}

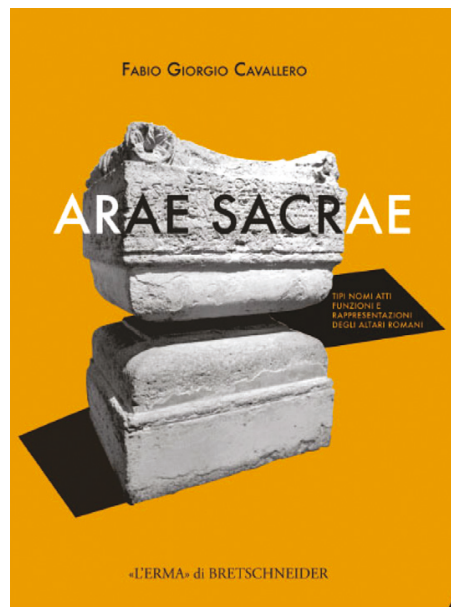

CAVALLERO, FABIO GIORGIO

(2018). Arae sacrae. Tipi, nomi, atti, funzioni e rappresentazioni degli altari romani. Roma: L’ Erma di Bretschneider. 368 pp., $342 €$ [ISBN 978-8-8891-3170-63].

\section{OLIVIER DE BIGAULT DE CAZANOVE Université PARIS-SorbonNe OLIVIER.DE-CAZANOVE@UNIV-PARIS1.FR}

LES AUTELS ROMAINS REVIENNENT AUJOURD' HUI au centre de l'attention, plus d'un siècle après le catalogue pionnier d'Helen Cox Bowerman (Roman sacrificial altars. An archaeological study of monuments in Rome, Lancaster, 1913) qui listait déjà 87 autels couvrant toute l'antiquité à Rome. On rappellera quelques étapes de cet intérêt pour ce qui est l'élément central du sanctuaire puisque c'est auprès de lui que s'accomplit le sacrifice : du côté grec, l'utile monographie de Constantine G. Yavis (1949); du côté romain, le livre d'Olaf Dräger sur le décor des autels de marbre (1994); et, en général, l'important colloque sur l'espace sacrificiel dans les civilisations méditerranéennes de l'antiquité (1991) que cependant F.G. Cavallero ne cite pas. Aujourd' hui donc paraissent presque simultanément deux livres dont l'objet est le même - les autels de Rome et du Latium antérieurs au principat - mais avec deux approches très différentes, qu'explicitent clairement leurs sous-titres respectifs. Quelques mois après le livre de Fabio Giorgio Cavallero est en effet sorti 
celui de Claudia Moser, The Altars of Republican Rome and Latium: Sacrifice and the Materiality of Roman Religion (Cambridge, 2019).

Le livre de F.G. Cavallero, comme beaucoup de publications actuelles de thèses de doctorat, peut être considéré comme une longue introduction (137 p.) au catalogue ou plutôt à plusieurs catalogues juxtaposés qui forment ensemble la deuxième partie de l'œuvre. Le premier catalogue est celui des « objets » eux-mêmes comme l'auteur les appelle, c'est-à-dire des 86 autels conservés d'époque archaïque et républicaine. Le deuxième catalogue liste 63 autels de Rome connus par les sources littéraires, cette fois-ci sans distinction de dates (on y trouve donc aussi bien l' Ara Maxima Herculis, considéré comme bien antérieur à la fondation de la Ville, ou bien l'autel de la piété plébéienne fondé en 296 av. J.-C. par Virginia, que l' Ara Pacis ou l'autel de Iuppiter Conservator dédicacé par Domitien). Le troisième regroupe les sources iconographiques, d'abord les monnaies (depuis le denier de L. Pomponius Molo, de 97 av. J.-C., avec Numa sacrifiant, jusqu'à des bronzes de Constantin, Crispus et Constantin II - et l'on aurait d'ailleurs aimé disposer, pour ce catalogue numismatique, d' un peu plus d'indications et de commentaires que de sèches références au $R R C$ et aux $R I C$ ), puis 14 fragments de la Forma Urbis Marmorea avec des représentations d'autels, enfin 55 reliefs (et une ciste de Préneste) avec des scènes sacrificielles à l'autel, qui proviennent de toute l' Italie. Ces catalogues sont indéniablement utiles, mais on mesure aussi leur hétérogénéité, en particulier chronologique. De quoi traite exactement ce livre auquel sa préface assigne un projet très général (« le arae sacrae... databili tra l' VIII secolo a. C. e il 391 d. C. ») ? Des autels romains en général ? De ceux du culte public ? Spécifiquement, de ceux de Rome et de ses alentours immédiats ? Plus spécifiquement encore, de ceux de l'époque archaïque et républicaine, comme le corpus de Realien sur lequel il se fonde le donne à penser ? Ce n'est pas diminuer la valeur de l'ouvrage, qui rendra certainement des services aux chercheurs, que de pointer le fait que son sujet est, en quelque sorte, à géométrie variable.

D' une autre manière, le livre de F.G. Cavallero ne se laisse pas enfermer dans des bornes sans doute trop restreintes. Son point de départ est clairement archéologique (les « objets ", c'est-à-dire les autels encore existants, envisagés dans leur matérialité, pour lesquels est proposée une typo-chronologie). Mais ensuite, on s'éloigne progressivement de ceux-ci, et les développements ultérieurs de la première partie n'y font plus du tout référence. C'est d'abord une enquête sur les noms par lesquels on désigne les autels, arae et altaria. F.G. Cavallero suit sur ce point un bref mais stimulant article de V. Gasparini ( Altaria o candelabra? Aspetti materiali del culto di Iside illuminati dalla testimonianza di Apuleio ", in L. Bricault éd., Bibliotheca Isiaca, I, Bordeaux, 2008, p. 39-47) dont F.G. Cavallero a repris et développé les conclusions dans une récente contribution à Archeologia Classica (68, 2017, pp. 589-601) : les 
altaria seraient formellement similaires à des candélabres ou à de hauts thymiateria, à ceci près que la flamme allumée au sommet de leur fût ne servirait pas seulement à éclairer ou encore à brûler de l'encens, mais aussi à consommer des offrandes végétales, non sanglantes (Serv., Georg. III 490) : parmi d'autres exemples, on peut citer une série de reliefs du forum de César où, entre deux putti (cependant tauroctones!), un pseudo-candélabre porte une coupelle où se consument fruits et pignes de pin. L' hypothèse Gasparini-Cavallero peut donc être acceptée, à condition sans doute d'admettre une certaine souplesse - d'ailleurs normale - dans le vocabulaire : les altaria pouvaient prendre cette forme et inversement les coupelles sur pied haut pouvaient être appelées ainsi. C'est ensuite un troisième chapitre sur les normes de droit sacré pour la construction et la dédicace d' un autel (votum, locatio, inauguratio, constitutio, consecratio, dedicatio) qui, dans la plupart des cas décalquent celles, mieux connues, pour l'établissement d' un temple, lorsque les autels ne sont pas, purement et simplement, compris dans les limites de celui-ci (lorsqu' ils s'élèvent sur l'escalier d'accès au podium) ou bien dans un espace consacré débordant son emprise stricte, auquel cas la dédicace de l'autel (et toute la série des opérations antérieures) est incluse dans celle du temple. Le chapitre suivant est une description de quelques éléments de la séquence sacrificielle, telle que les sources textuelles - et secondairement iconographiques - permettent de la reconstituer (une référence à la récente synthèse du ThesCRA I eût été bienvenue). Les deux derniers brefs chapitres (l'autel et le serment, l'autel et le droit d' asile) reviennent sur ces questions de droit qui sont l'objet d' un intérêt particulier de la part de l'auteur, comme le montre d'ailleurs le reste de sa bibliographie.

Revenons à la typologie des autels républicains, qui constitue le cœur de ce travail. Deux groupes fondamentaux sont distingués : les autels « a profili contrapposti » (c'est-à-dire en forme de sablier) et ceux « à corps lisse » (qui, en réalité, présentent eux aussi des corniches inf. et sup., mais séparées par une zone médiane verticale, portant parfois une inscription). Le premier groupe est ensuite subdivisé : autels à antes (c'est-à-dire en $\mathrm{Pi}$ ), qui apparaissent dès le milieu, voire dans la première moitié du $\mathrm{VI}^{e} \mathrm{~s}$. av. J.-C., et autels sans antes, qui sont plus tardifs (à partir du IV s. ; pour l'auteur en effet, l'autel tardo-archaïque de Corcolle était du type à antes). Contrairement à l' Étrurie, où la variabilité des formes est plus grande, l'autel en Pi à profil en forme de sablier est donc l'autel latin par excellence entre le $\mathrm{VI}^{\mathrm{e}}$ et le IV $\mathrm{I}^{-} \mathrm{III}^{\mathrm{e}}$ s. av. J.-C. À vrai dire, à cette prédominance écrasante (sinon cette exclusivité), concourt largement la série des autels de Lavinium. Mais appartiennent aussi à ce groupe, à Rome, le Volcanal et les autels de S. Omobono ; à Ardée, ceux de la Banditella et l'autel in antis du Fosso dell' Incastro ; ainsi que ceux de Mentana, de Tivoli et de Corcolle. Si l'autel traditionnel est celui en Pi, on comprend mieux 
le recours à cette forme spécifique à l' intérieur de l'enclos de l'ara Pacis, avec certes aussi d'autres modèles, qui sont plus directement grecs.

Dans le sous-groupe des autels en $\mathrm{Pi}$, deux variantes sont distinguées par l'auteur : avec ou sans marches entre les antes. Il eût peut-être mieux valu prendre en considération la technique d'assemblage des bases d'autels, qui a fait l'objet d'une classification typologique de C.F. Giuliani et P. Sommella (PP, 32, 1977, p. 360, fig. 3 , à partir de la documentation de Lavinium), récemment reprise et élargie par D.P. Diffendale (in P. Brocato, M. Ceci, N. Terrenato éd., Ricerche nell'area dei templi di Fortuna e Mater Matuta. 2016, p. 150, fig. 8, qui distingue "Lavinium box-type ", " Lavinium platform-type », et " Fosso dell' Incastro type ». Et cela, d'autant plus que cette classification est porteuse d' informations chronologiques : le type " a scatola " (parements de blocs et noyau central) serait le plus ancien (mi VI ${ }^{\mathrm{e}}$-fin IV s. av. J.-C.). On fera une autre remarque sur la chronologie : celle de l'autel XII de Lavinium, généralement daté - y compris par l'auteur - de la fin du IV s., doit être abaissée à la première moitié du III , comme l'indique une coupelle à vernis noir (série 2783 ou 2787 Morel) scellée à l' intérieur de l'autel. Les profils des podiums des temples de Sora (col. lat. 303), de Villa S. Silvestro (après la conquête de la Sabine, en 290) et d' Isernia (col. lat. 263) sont d'ailleurs similaires à celui de l' autel XII.

Dernière remarque sur les autels en $\mathrm{Pi}$ : ils permettent précisément de connaître l'orientation de l'autel et du sacrifiant, puisque celui-ci se tenait entre les antes. Tous les autels sont - comme on pouvait s'y attendre - orientés vers l'est, à l'exception du Volcanal, étrangement orienté au sud-ouest (et non au nord, comme indiqué sur la pl. $13 \mathrm{~A}$ ). Ce n'est d'ailleurs pas le seul caractère atypique de cet autel, dont les antes sont disproportionnées par rapport à l'élément central, réduit à une unique file de blocs. L'orientation des autels au levant explique que l'officiant pouvait être amené à sacrifier perpendiculairement à l'axe du temple : ainsi en estil, à Rome, à S. Omobono ; ou à Ardée, au temple B du Fosso dell' Incastro. Plus tard, lorsque les autels « à corps lisse » auront pris le relais, celui de Junon à Gabies (vers 160 av. J.-C.) se dispose de manière identique pour faire face à l'est, un de ses côtés courts regardant vers le temple (et on pourrait ajouter qu' il en est de même à Pompéi, au temple d'Apollon, au temple dit d' Esculape, etc.).

Entre les autels à antes et les autels " à corps lisse " s' intercale le groupe A2 de l'auteur, c'est-à-dire les autels « a profili contrapposti » mais sans antes. Ils sont beaucoup plus petits que les précédents, et - à une exception près, sur laquelle je vais revenir - de plan carré. Il me semble donc qu' il existe entre eux et le groupe des autels à antes la même différence que le Dictionnaire méthodique de l'architecture grecque et romaine de R. Ginouvès pose (III, 1998, p. 49-50) entre les « autels monolithiques » et les « autels construits ", à ceci près que les autels du groupe A2 sont « a dadi sovrap- 
posti », c'est-à-dire fait de deux blocs (ou plus) superposés. Quant aux autels «à corps lisse ", subdivisés en rectangulaires (B1), carrés (B2) et ronds (B3), il est clair qu'à nouveau les deux dernières variantes correspondent à des autels monolithiques, et conviennent à des lieux de culte mineurs, au moins par les dimensions (ex. : le temple A du Fosso dell' Incastro, daté du II ${ }^{e}$ s. av. n. è.), tandis que les autels rectangulaires sont des autels construits, qui conviennent à des grands temples. Ceux à frise dorique (Junon à Gabies, Esculape à Pompéi ; cf. le sarcophage de Scipion Barbatus) sont particulièrement connus. Ils datent du $\mathrm{II}^{\mathrm{e}} \mathrm{s}$. av. J.-C., alors que certains autels carrés « à corps lisse » remontent au siècle précédent.

Cette claire sériation chronologique et fonctionnelle permet, me semble-t-il, de dire quelque chose sur un cas isolé, qui rentre mal dans la typologie proposée par l'auteur : l' unique autel "a profili contrapposti ", sans antes, rectangulaire (et non carré). Il se trouve devant l'escalier d'accès au podium du temple B du Fosso dell' Incastro près d'Ardée. Il présente indéniablement la même moulure de base que l'autel en Pi situé juste à côté, mais il est orienté différemment. Cette différence d'orientation est expliquée, dans la récente publication (2018) de Castrum Inui (M. Torelli, E . Marroni éd.) par des raisons rituelles : on aurait là la reproduction des autels " troyens " mentionnés par Denys d' Halicarnasse (I, 55, 2), «l'un tourné vers le levant, l'autre vers le couchant ", élevés par Énée à l'endroit où il avait débarqué dans le Latium, pour honorer le Soleil. Cette hypothèse est certes séduisante, et la contemporanéité des deux autels semblerait garantie par la ressemblance de leur moulure de base. Et pourtant, l'autel sans antes est postérieur, comme le montre sans doute possible le fait qu' il «mord » sur le dallage entourant l' autel en Pi (p. 26, pl. XX et Castrum Inui, p. 125, fig. 46). Comme son assise inf. n'est faite que de deux blocs juxtaposés, on pourrait le considérer, lui aussi, comme une structure " a dadi sovrapposti », plutôt que comme un autel véritablement construit. On pourrait le considérer également comme une forme de transition entre les autels à antes des $\mathrm{VI}^{\mathrm{e}}-\mathrm{III}^{\mathrm{e}}$ s., et les autels

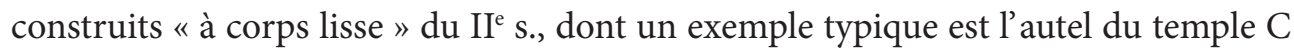
du Largo Argentina, dédicacé par A. Postumius Albinus.

L'ouvrage de F.G. Cavallero sur les Arae sacrae est donc l' un de ces livres qui devraient permettre aujourd' hui de "revenir à l'autel ", à partir des objets eux-mêmes c'est-à-dire des structures archéologiquement attestées, étudiées en contexte -, alors que bien souvent, on se contente, pour restituer la séquence sacrificielle, de combiner images et textes. En ce sens, on pourrait l'insérer dans un filon d'études, celui de l'Archaeology of Religion in the Ancient World, pour reprendre le titre d'un récent Companion édité par R. Raja et J. Rüpke (2015). Dans ce domaine, beaucoup reste à faire (par exemple, l'examen tracéologique des signes laissés sur les parements, les marches, la table d'autel par un usage répété ; ou encore l'étude archéozoologique, 
mettant en rapport ossements animaux et structures d'autel - malgré le fait que les sanctuaires antiques, à la différence de ceux de la Gaule par exemple, étaient périodiquement nettoyés). En amont de ces nouvelles approches de l'espace sacrificiel, dont il faut souhaiter le développement, un préalable indispensable est le classement typo-chronologique des autels. Celui qui est proposé ici, et qui structure aussi le catalogue des 86 autels pris en considération, est assurément une œuvre utile, comme l'est le livre à la visée plus vaste dans lequel il s' insère. 